\title{
INTERVENÇÕES DA TERAPIA COGNITIVO-COMPORTAMENTAL (TCC) PARA TRANSTORNO DE ESTRESSE PÓS-TRAUMÁTICO: ESTUDO DE CASO
}

INTERVENTIONS OF COGNITIVE-BEHAVIORAL THERAPY (CBT) FOR POST TRAUMATIC STRESS DISORDER: A CASE STUDY

\section{Gabriela Maffini', Paula Argemi Cassel ${ }^{2}$}

RECEBIDO EM: 28/02/2020 | ACEITO EM: 18/05/2020

DOI: $10.5902 / 2317175842659$

\section{RESUMO}

O presente trabalho objetiva apresentar técnicas de Terapia Cognitivo-Comportamental (TCC) para sintomatologia de Transtorno de Estresse Pós-Traumático (TEPT), utilizadas em atendimento psicológico, em serviço-escola, de uma jovem adulta de 19 anos, vítima de abuso sexual intrafamiliar. Trata-se de uma pesquisa qualitativa de estudo de caso único. Como resultado, constatou-se efetividade ao longo do tratamento, considerando a sintomatologia inicial e final, bem por observações clínicas e avaliações de testes psicológicos. Utilizam-se técnicas da TCC, como a reestruturação cognitiva, exposição prolongada do trauma e técnicas de relaxamento, para promoção do bem-estar e manejo de sintomas que causassem prejuízo funcional. Entende-se que apesar de ser evidenciado progressos no tratamento, se faz necessário que a paciente dê continuidade ao processo de ressignificar experiências relacionadas ao abuso sexual que vivenciou durante grande parte de sua infância e adolescência.

Palavras-chave: Transtorno de Estresse Pós-Traumático; Terapia Cognitivo Comportamental; Abuso sexual intrafamiliar; Estudo de caso. 


\begin{abstract}
The present work aims to present techniques of Cognitive-Behavioral Therapy (CBT) for symptoms of Posttraumatic Stress Disorder (PTSD), used in psychological care, in school service, of a young adult of 19 years, victim of intrafamiliar sexual abuse. This is a qualitative study of a single case stu$d y$. As a result, effectiveness was found throughout the treatment, considering the initial and final symptoms, as well as clinical observations and psychological test evaluations. CBT techniques are used, such as cognitive restructuring, prolonged exposure to trauma and relaxation techniques, to promote well-being and manage symptoms that cause functional impairment. It is understood that despite progress in treatment, it is necessary for the patient to continue the process of reframing experiences related to sexual abuse that she experienced during much of her childhood and adolescence.
\end{abstract}

Keywords: Posttraumatic Stress Disorder; Cognitive behavioral therapy; Intrafamily sexual abuse; Case study.

\title{
1 Introdução
}

A exposição ao trauma pode ocasionar no indivíduo o desenvolvimento de um conjunto de transtornos, os quais ocasionam sofrimento psicológico cotidiano intenso, tornando difícil a tarefa de desempenhar ações que, até determinado momento de suas vidas, eram consideradas simples (WHITBOURNE; HALGIN, 2015). Os transtornos relacionados a trauma e a estressores incluem transtornos nos quais o sujeito passou por exposição a um evento traumático, seja de forma direta ou indireta, podendo se classificar em transtorno de apego reativo, transtorno de interação social desinibida, transtorno de estresse agudo, transtorno de estresse pós traumático (TEPT) e transtornos de adaptação (APA, 2014).

O TEPT foi introduzido pela primeira vez como transtorno no Manual diagnóstico e estatístico de transtornos mentais, em sua terceira edição (DSM-III), no ano de 1980. Antes desta classificação, a nomenclatura utilizada para pessoas que vivenciavam sintomas de estresse consistia em "neurose traumática" ou "neurose de guerra", em razão do estudo de pesquisadores sobre a manifestação de sintomas comuns á aqueles que experienciaram traumas, ser desenvolvido e aprofundado com o público-alvo de ex-combatentes de guerra, em especial, da Guerra do Vietnã e pessoas que vivenciaram o trauma relacionado a catástrofes naturais (SILVA, 2017).

Consta-se na publicação do DSM-V que a característica principal do TEPT é o desenvolvimento de sintomas característicos após a exposição ao trauma como ameaça de morte, lesão grave ou violência sexual em uma das seguintes formas: vivência direta, testemunhar pessoalmente o evento traumático com outras pessoas, saber que o evento traumático ocorreu com alguém próximo ou ser exposto de forma repetida a detalhes do trauma (APA, 2014). Dentre as situações desencadeadoras de TEPT, estudos indicam que este transtorno, em crianças e adolescentes, é mais comum diante da exposição e/ou vivência de abuso sexual (RUGGIERO; MCLEER; DIXON, 2000; RUNYON; KENNY, 2002). 
Ademais, é necessária a presença de um ou mais de sintomas intrusivos como lembranças angustiantes constantes, sonhos recorrentes nos quais o conteúdo/sentimento está associado ao trauma, reações dissociativas (nas quais o sujeito sente ou age como se o trauma estivesse ocorrendo novamente), sofrimento psicológico intenso ou prolongado ante a sinais que simbolizem a experiência e reações fisiológicas. Para a classificação deste transtorno podem ser evidenciadas evitação persistente de estímulos que relembrem o trauma, alterações cognitivas e no humor, alterações marcantes na excitação e reatividade, apresentando então comportamento irritadiço e surtos de raiva, comportamento imprudente e autodestrutivo, hipervigilância, resposta de sobressalto exagerada, perturbação no sono, causando significativo prejuízo funcional em importantes áreas da vida do sujeito (APA, 2014). Para receber a classificação de TEPT, existe o critério dos sintomas prolongarem-se por um período maior que de um mês, visto que períodos inferiores a estes enquadram-se na classificação de transtorno de estresse agudo (SILVA, 2017; CLARK; BECK, 2012).

Para o desenvolvimento infantil e adolescente, considera-se o abuso sexual como um grande fator de risco devido à possibilidade de existirem consequências emocionais, cognitivas e comportamentais. O abuso sexual na infância e adolescência é definido como uma exploração com cunho sexual, sendo ocasionado por uma pessoa que esteja em estágio psicossexual mais avançado do que a vítima. Destaca-se que o abuso pode ser classificado como intrafamiliar ou extrafamiliar, sendo o primeiro caracterizado por quando o abusador encontra-se na mesma família e o extrafamiliar, caracterizado pelo abusador não ser da mesma família. Diante do abuso intrafamiliar, há maior dificuldade em ocorrer denúncias, visto que há o receio de modificações no ambiente familiar (BRINO; WILLIANS, 2003; SIQUEIRA; ARPINI; SAVEGNAGO, 2011).

Dentro da situação de abuso, pode-se haver o uso de força física por parte do agressor, assim como o uso de ameaças que interfiram no bem-estar físico e emocional da criança, assim como na possibilidade de ferir seus entes queridos, o uso de mentiras, assédio físico e moral, exposição genital, uso de pornografia e relações com penetração. $\mathrm{O}$ uso de coerção, impossibilidade de decisões por parte da criança ou adolescente e o uso da vítima para satisfação sexual exclusiva do agressor, se fazem comuns aos conceitos de abuso sexual (PADILHA; GOMIDE, 2004). Entretanto, compreende-se que os casos de abuso sexual apesar de possuírem elementos em comum são distintos entre si. Logo, cada indivíduo possui diferentes necessidades para serem atendidas na psicoterapia (FLORENTINO, 2014).

Frente aos marcos que a vivência de abuso gera no indivíduo, aponta-se para a importância do sujeito ser amparado e acolhido, inserindo-se, assim, a importância do papel da Psicologia, qual poderá ajudar o indivíduo a ressignificar o acontecimento, recuperar experiências e estar aberto a novas vivências. A psicoterapia de orientação cognitiva-comportamental é apontada como eficaz na redução de sintomas comuns as vítimas de abuso, auxiliando a lidar com 
os sentimentos e emoções provenientes do(s) evento(s) traumático(s) (CLARK; BECK, 2012; DURAN, 2015).

A terapia cognitivo-comportamental (TCC) é considerada como superior na intervenção com pacientes expostos a violência sexual e no manejo de sintomas provenientes do Transtorno de estresse pós-traumático, por apresentar resultados significativos em comparação a outras abordagens (HABIGZANG; CAMINHA, 2004 ; LUCÂNIA, et. al, 2009; POWERS, et. al, 2010; CLARK; BECK, 2012; MONSON; RESICK; RIZVI, 2016, OLIVEIRA, 2017). Como evidência, a TCC tem sido amplamente recomendada como tratamento indicado em diretrizes de tratamento para o TEPT (NATIONAL INSTITUTE OF CLINICAL EXCELLENCE, 2005).

A predominância da TCC como tratamento recomendado é associada a determinadas características desta abordagem, referentes ao caráter objetivo e focado no presente, além de garantir a participação ativa do paciente durante o processo terapêutico, existindo uma relação de colaboração entre o terapeuta e o paciente, os quais, juntos, operam como uma equipe (BECK, 2013). A TCC, inicialmente desenvolvida por Aaron Beck como um modelo de tratamento para depressão, vem ganhando reconhecimento sobre a efetividade de resultados diante do acompanhamento de diversas psicopatologias, entre elas o TEPT (MELLO, et. al, 2011; OLIVEIRA, 2017).

Os efeitos da TCC consistem em, a partir da relação de colaboração entre o terapeuta e o paciente, auxiliar na identificação de crenças disfuncionais e auxiliar na reestruturação cognitiva do paciente(BECK, 2013). Mais especificamente, no tratamento para o TEPT, provocado pela exposição ao abuso sexual, a TCC visa auxiliar na modificação de crenças e sentimentos negativos relacionados à ocorrência do abuso, assim como utilizar técnicas como exposição gradual, intencionando a evocação de memória e conteúdos traumáticos. Os conteúdos evocados podem auxiliar na redução de sintomas do TEPT, pelo uso de técnicas de enfrentamento, coping e da psicoeducação do paciente sobre o transtorno (PASSARELA; MENDES; MARI, 2009)

Este artigo tem como objetivo apresentar um estudo de caso, qual relata o atendimento psicológico embasado pela TCC, realizado com uma paciente jovem do sexo feminino, que por sua vez apresentava sintomas de TEPT, em decorrência de ter vivenciado abuso sexual intramiliar. Ainda, este trabalho visa maior compreensão sobre o TEPT e sua sintomatologia.

\section{Metodologia}

Este trabalho trata-se de uma pesquisa qualitativa de cunho exploratório e descritivo, cuja análise foi baseada em teorias, buscando por evidências para sustentar os achados teóricos, com vistas a encontrar um resultado que identifique a existência de padrões entre as informações coletadas (DENZIN; LINCOLN, 2000; SILVA, HERZBERG; MATOS, 2015). Utilizou-se de estudo de caso único para o desenvolvimento visto que este busca a investigação de uma 
realidade, a fim de compreensão total e transformação no conteúdo obtido em escrita científica (COIMBRA; MARTINS, 2008).

Os atendimentos foram realizados dentro de um laboratório de práticas em Psicologia, localizado em uma universidade particular no interior do Rio Grande do Sul. A paciente, Priscila (nome fictício a fim de preservar todos os envolvidos) foi atendida semanalmente durante o período de oito meses, havendo um mês de recesso devido às férias universitárias, totalizando 23 atendimentos psicológicos com a paciente, além de dois atendimentos com a mãe e dois atendimentos com o pai de Priscila.

A paciente foi atendida por uma estagiária do quarto ano do curso de Psicologia, a qual semanalmente escrevia relatórios, a fim de levar os dados colhidos nos atendimentos para supervisão, em que eram discutidos os acontecimentos de cada sessão. As escolhas da intervenção definiram-se devido a objetivos específicos, sendo utilizadas técnicas fundamentadas pela teoria cognitivo-comportamental. Para verificação de humor da paciente, optou-se por utilizar do instrumento de testagem psicológica, o Inventário de Depressão de Beck (BECK; STEER; BROWN, 2011). Já em sessões espaçadas e para avaliar questões relacionadas à dificuldade de aprendizagem da paciente utilizou-se o teste a Escala Wechsler de Avaliação Breve de Inteligência (WECHSLER, 2014). Para assegurar o sigilo profissional e permitir o uso de seus dados para estudos, a paciente assinou o termo de consentimento de uso de dados de prontuários e pesquisa .

\subsection{Instrumentos}

Salienta-se que a aplicação do Inventário de Depressão de Beck (BECK; STEER; BROWN, 2011) é utilizada no atendimento clínico devido ao fato de ser considerada uma das melhores escalas de autorrelato de depressão. $O$ teste, atualmente, encontra-se em sua segunda edição, o BDI-II, o qual foi desenvolvido para superar questionamentos sobre sua validade e fidedignidade. $\mathrm{O}$ teste possui 21 itens de avaliação da severidade da sintomatologia, divididos em afirmações que devem ser classificadas entre 0-3 pontos, possuindo como pontos de corte de 0-13 pontos a classificação de depressão mínima, entre 1419, depressão leve, entre 20-28, depressão moderada e resultados acima de 28 são considerados como depressão severa (BECK; STEER; BROWN, 2011).

O uso do teste encontra-se favorável pelo Conselho Federal de Psicologia (CFP) e foram realizados diversos estudos para a validação do teste. Dessa forma pode-se citar a pesquisa desenvolvida no Rio Grande do Sul - RS, realizada em escolas públicas e privadas da capital com adolescentes entre as idades de 13-19 anos de idade, totalizando 391 estudantes, do sexo masculino e feminino, possuindo como resultado que a estimativa de fidedignidade, baseada no Coeficiente Alfa de Cronbach neste estudo, de 0.86 , considerado satisfatório (PARANHOS, 2009). Optou-se por aplicar este instrumento, para auxiliar na 
avaliação da efetividade da psicoterapia para os sintomas de TEPT da paciente, assim como a efetividade do medicamento prescrito pelo psiquiatra.

Em relação à aplicação da Escalas Wechsler de Avaliação Breve de Inteligência (WECHSLER, 2014), esta foi desenvolvida como uma escala independente, formada pelos subtestes de Vocabulário, Cubos, Semelhanças e Raciocínio Matricial, quais foram selecionados devido à associação com habilidades cognitivas gerais e pela representação de habilidades verbais e de execução (THE PSYCHOLOGICAL CORPORATION, 1999). O subteste do Vocabulário apresenta 4 itens representados por figuras e 38 itens representados por palavras. Já no subteste dos Cubos, o examinando utiliza de 9 cubos para produzir até 13 figuras, havendo tempo limite para cada figura. O subteste das Semelhanças tem como objetivo identificar figuras semelhantes nos primeiros quatro itens ou explicar como dois conceitos se assemelham, enquanto no subteste de Racíocino Matricial, o examinando deve completar uma figura entre as opções, afirmando qual está faltando. Entre estes subtestes, existem critérios de interrupção para erros consecutivos. (YATES, et. al, 2006; WECHSLER, 2014).

Este instrumento é aplicado de forma individual, para indivíduos entre as idades de 6-89 anos, sendo avaliados os aspectos cognitivos como o conhecimento verbal, o processamento de informações visuais, inteligência fluida e cristalizada, raciocínio espacial e não verbal. Os quatro subtestes fornecem o QI da escala total, enquanto os subtestes de Vocabulário e Semelhancas fornecem o QI verbal e os subtestes de Cubos e Racíocinio Matricial, o QI de execução (THE PSYCHOLOGICAL CORPORATION, 1999). Para estabelecer a fidedignidade dos substestes, utilizou-se da fórmula Spearmann-Brown, de forma que obteve-se como resultado uma variação entre os coeficientes de precisão, sendo eles, entre 0,81-0,97 para crianças e para adultos 0,84 e 0,97, sendo considerado um resultado satisfatório (YATES, et. al, 2006). Este teste foi utilizado com a finalidade de avaliar as funções cognitivas da paciente e obteve como resultados a constatação de que, a partir dos dados normativos da escala, a paciente apresentou inteligência geral entre médio inferior e médio, considerando-se o desvio padrão.

\section{Resultados e discussões}

Priscila é uma jovem do sexo feminino, com 19 anos de idade na data em que se realizaram os atendimentos psicológicos. É a filha mais nova de casal divorciado, sendo que possui uma irmã por parte de mãe e é filha única por parte de pai. Os pais se divorciaram quando estava no início da adolescência, sendo o casamento marcado por violência física e psicológica. Estudou a vida toda em escola pública e no início do percurso escolar, Priscila não apresentou dificuldades, entretanto, após chegar no ensino médio, acabou repetindo o primeiro ano.

A fase inicial de seu desenvolvimento deu-se dentro do núcleo familiar parental, em que a avó paterna se fazia bastante presente, além da família do 
tio também, pois moravam no mesmo terreno de sua residência. Segundo relato da paciente, desde os seus 6 até os 14 anos, sofreu abuso sexual por parte do tio paterno. Evidencia-se que aos 14 anos houve mudança de residência e endereço, permitindo que a situação de abuso se interrompesse.

O seu núcleo familiar pertence a um nível socioeconômico considerado baixo pelo IBGE, situação que dificultou assegurar as necessidades formativas de Priscila. Os pais, Vera e Lúcio (nomes fictícios), não desconfiavam de qualquer forma de abuso que a filha viesse a sofrer, tendo o tio (irmão do pai) como figura de confiança. Priscila acabava frequentando diversas vezes a casa do tio, com razões de visita ou a pedido dos familiares, sendo vítima de agressões psicológicas, físicas, estupro, uso de ameaças e mentiras para que não contasse a ninguém o que ocorria.

A paciente foi encaminhada para o laboratório de práticas em Psicologia por indicação do núcleo de práticas jurídicas (NPJ) da mesma universidade. Ao serem iniciados os atendimentos psicológicos, a paciente deixou claro que estava aliviada por ser atendida por uma estagiária do sexo feminino, visto que não conseguiria ficar sozinha em uma sala com alguém do sexo masculino. De acordo com Clark e Beck (2012), indivíduos com TEPT desenvolvem esquemas negativos sobre o mundo, incluindo sobre outras pessoas.

Nas sessões iniciais, a paciente não apresentou expressões ou comportamentos de angústia, relatando os episódios do abuso sexual vivenciados com uma expressão de tranquilidade. Acredita-se que isto tenha ocorrido pela paciente apresentar-se despersonalizada e desconexa, pois utilizava a mesma expressão facial, independente do conteúdo relatado por si. A dissociação, entorpecimento emocional e supressão deliberada de emoções frequentemente estão presentes no TEPT, sendo evidenciado um "torpor psíquico', onde há anulação de sentimentos de prazeres, os quais foram sufocados pelo trauma (SILVA, 2017).

Para ocorrer o diagnóstico de TEPT, de acordo com a Associação Psiquiátrica Americana - APA (2014), o indivíduo deve atender os seguintes critérios: Critério A - exposição a episódio concreto de ameaça de morte, lesão, ou abuso sexual em uma das seguintes formas: vivenciar diretamente o evento; testemunhar o evento ocorrido com outras pessoas; saber que ocorreu com familiar ou amigo próximo; ser exposto repetidamente a detalhes do trauma. Ao ser investigado, constata-se que além de ter vivenciado por um período de aproximadamente nove anos de abuso sexual, primas de Priscila também vivenciaram a mesma situação, enquadrando-se também na subcategoria de saber que ocorreu com familiares próximos. O tipo de trauma, sua gravidade e a resposta emocional variam de acordo com o envolvimento pessoal do sujeito (SILVA, 2017).

Ademais, existe a necessidade de se fazer presente um ou mais dos seguintes sintomas para satisfazer o critério B: lembranças intrusivas angustiantes e involuntárias do trauma, sonhos angustiantes que estejam associados ao trauma, reações dissociativas (flashbacks) através dos quais o sujeito sente que está vivenciando novamente o trauma, sofrimento psicológico intenso diante de 
sinais que simbolizem o evento traumático e reações fisiológicas intensas (APA, 2014). Após os atendimentos iniciais, a paciente relata que além da sua desconfiança com pessoas do sexo masculino, ocorreu, em duas situações de tentativa de suicídio, ver o tio no lugar dos colegas que tentavam a ajudar, sentindo que estava revivendo a violência sexual. A manifestação de flashbacks é caracterizada pela sensação da vítima de estar revivendo o evento traumático independentemente do local que esteja inserida, sendo desencadeado por gatilhos que a relembrem da ocasião traumática (GADINI; JÚNIOR; FEIJÓ, 2018).

O critério $C$ envolve a evitação de pensamentos, recordações e eventos, assim como de pessoas, lugares, conversas e objetos associados ao trauma. Já o critério $D$ envolve a alteração negativa tanto na cognição quanto no humor, como incapacidade de recordar algum aspecto importante do trauma; desenvolver crenças negativas persistentes sobre si e sobre outros, estado emocional negativo persistente; interesse e participação diminuída, sentimentos de distanciamento e alienação em relação aos outros, enquanto o critério $E$ envolve apresentar alterações marcantes na excitação e na reatividade associadas ao trauma, apresentando pelo menos dois aspectos: comportamento irritadiço e surtos de raiva, comportamento imprudente e/ou autodestrutivo, hipervigilância, problemas de concentração, perturbação no sono (APA, 2014).

$\mathrm{Na}$ terceira sessão de atendimento, Priscila relata estar com dificuldade de dormir, dormindo uma pequena quantia de horas por noite e logo acordando, além de estar apresentando respostas de sobressalto perante barulhos excessivos e definir que seu comportamento em casa está mais agressivo em relação a seus familiares, principalmente; ainda, apresentava alteração cognitiva e do humor, bem como sofrimento psicológico intenso, com reações fisiológicas. Outro aspecto é que a paciente evitava estímulos que remetessem ao trauma, como sons altos, como gritos de pessoas. A partir da constatação que estes sintomas fazem parte do TEPT, a paciente foi encaminhada para atendimento psiquiátrico, de forma que em conjunto com a psicoterapia, também fosse assistida psiquiatricamente. $O$ tratamento medicamentoso é feito com o uso de substâncias que ajudam a regularizar os níveis de neurotransmissores cerebrais, como os Inibidores Seletivos da Recaptação da Serotonina (ISRS), Inibidores Seletivos da Recaptação da Serotonina e Noradrenalina (IRSN), antidepressivos tricíclicos e alguns ansiolíticos (SILVA, 2017).

Posteriormente, após a constatação dos sintomas de TEPT, na quarta sessão de atendimento, iniciou-se a psicoeducação com a paciente e foram feitas sessões de psicoeducação com sua mãe e seu pai (separadamente), de forma que os sintomas e condições psicológicas que a paciente apresentava fossem compreendidos, assim como a importância de haver a continuidade semanal da psicoterapia e de haver o encaminhamento psiquiátrico, visto que a medicação seria uma forma de auxílio no manejo dos sintomas. As sessões da TCC focalizam a educação do paciente sobre o transtorno, fornecem informações cognitivas para a manutenção dos sintomas pós-trauma, a apresentação da justificativa lógica do tratamento e os objetivos para este (DIAS; CANAVEZ; MATOS; 2018). 
O encontro com a mãe trouxe preocupação para a estagiária, visto que a mãe, ao ser chamada pelo nome na sala de espera pela estagiária, entrou, juntamente a ela na sessão, um homem, o qual a estagiária questionou sobre a relação entre os dois, tendo a mãe respondido que não havia nenhuma e não se conheciam, tendo este homem se confundido nas salas de atendimento. Entretanto, a mãe não apresentou nenhuma resistência sobre a presença dele, falando sobre coisas pessoais suas. Dando continuidade ao mesmo atendimento, a estagiária explicou que a mãe havia sido chamada para conversar sobre o quadro sintomático de TEPT que Priscila estava apresentando, tendo a mãe hesitado e afirmado que estava ali pela sua outra filha, de nome Pâmela, demorando alguns segundos para corrigir-se. Lúcia não demonstrou reações emocionais, tendo dificuldade em falar sobre o caso e sobre a filha, relatando que a esta se recusa a falar sobre com ela. Quando há abuso intrafamiliar, envolve a quebra de confiança com as figuras parentais, uma vez que era de sua responsabilidade promover a segurança física e psicológica da criança e/ ou adolescente, sendo assim, considera-se que a ausência de disponibilidade de escutar os filhos gera um sentimento de desamparo neles (DE ANTONI; KOLLER, 2002; SIQUEIRA; ARPINI; SAVEGNAGO, 2011).

No encontro com o pai, este demonstrou ser bastante presente na vida da filha e preocupado, questionando a estagiária sobre as melhores formas de auxiliar a filha, além de dar informações relevantes sobre o seu desenvolvimento e proporcionar um entendimento maior sobre o funcionamento familiar. Além disso, pai e mãe foram orientados sobre a preocupação de Priscila vir a se machucar, tendo em vista que apresenta pensamentos disfuncionais e comportamento autodestrutivo, sendo orientado maior atenção e cuidado para prevenção. Para o diagnóstico do TEPT, é comum a apresentação de alterações marcantes na excitação e na reatividade associadas ao evento traumático, como comportamento imprudente ou autodestrutivo (APA, 2014).

Para se ter uma maior compreensão do quadro depressivo que a paciente apresentava, utilizou-se o Inventário de Depressão de Beck (BECK; STEER; BROWN, 2011), que consiste em um instrumento de testagem psicológica, composto por 21 itens, utilizado com a finalidade de mensurar a severidade da sintomatologia depressiva apresentada pela paciente, acompanhando o progresso obtido pelo conjunto da psicoterapia com as sessões psiquiátricas. Este manual apresenta, como classificação, pontos de corte para avaliação da intensidade dos sintomas depressivos, sendo eles divididos em pontuação mínima (0-13), leve (14-19), moderada (20-28) e grave (29-63) (BECK; STEER; BROWN, 1996).

No total, o teste foi aplicado três vezes, com um intervalo de tempo em média de um mês. Na primeira aplicação a paciente pontuou 44 pontos, enquadrando-se no quadro de depressão grave. Evidenciava-se sintomas quais colaboraram com o resultado como tristeza, mudança de humor/comportamento, dificuldade para dormir, falta de apetite e pensamentos suicidas. A segunda aplicação obteve-se uma pontuação de 30 como o total, sendo ob- 
servadas pequenas mudanças realizadas em seu cotidiano, principalmente durante as verificações de humor, ainda que apresentasse sintomas depressivos e dificuldades de estabelecer relações. Já na terceira aplicação do teste, em decorrência de mudanças em sua rede de apoio, esperava-se que o resultado do teste se mantivesse o mesmo ou a alteração fosse mínima, obtendo-se a pontuação de 32. Para a avaliação da evolução da sintomatologia dos pacientes considera-se o Inventário de Depressão de Beck como uma ferramenta importante (SILVA; WENDT; ARGIMON, 2018).

No ambiente familiar de Priscila, os pais evitavam o assunto, todavia, devido à necessidade da paciente falar a respeito para que o processo de ressignificação pudesse ocorrer, recomendou-se fortemente a permitirem-se conversar com a filha, para auxiliar Priscila ao manejo de seus pensamentos e emoções. Ser capaz de falar sobre o trauma é um passo crítico no processo terapêutico, no qual recomenda-se que a vivência do paciente não seja reprimida, para que este consiga dar novos significados as suas memórias traumáticas (SILVA, 2017).

Iniciou-se, então, este processo no qual utilizamos desenhos, pois a paciente apresentava esta preferência. Nestes atendimentos, foram desenhados dois armários, classificando-os em armários com lembranças boas e armários com lembranças ruins. O tempo de duração da atividade variava de acordo com a limitação da paciente, pois se era pedido que atribuísse uma nota de 1 a 10 às lembranças ruins para se ter uma noção de quanto seria permitido continuar. As técnicas da TCC permitem ressignificar as experiências para diminuição da sintomatologia do TEPT, utilizando a modificação da recordação do trauma por meio da exposição em imaginação repetida, discussão verbal e questionamento socrático (CLARK; BECK, 2012).

Nas próximas sessões, usufruímos de folhas de desenho para que a paciente relatasse as situações vivenciadas. Priscila desenhou várias ocasiões e, pela estagiária, era requisitado que a paciente se colocasse nas situações novamente, falando de sentimentos, sensações, acontecimentos, odores ou até mesmo lembranças de como encontrava-se o clima. Evidenciava-se nestas sessões que Priscila encontrava-se despersonalizada, apresentando uma postura de como se não houvesse ocorrido com ela, e sim com outra pessoa, que a havia relatado. A dissociação e despersonalização são fatores comuns a pessoas com sintomas de TEPT (APA, 2014).

Ademais, trabalhou-se com a reestruturação cognitiva, qual envolve a identificação e modificação de crenças relacionadas ao trauma, principalmente em relação a generalização e dificuldade de confiar em pessoas do sexo masculino e de conseguir ficar perto dos colegas de aula, fato que fazia com que a paciente ficasse a maior parte do tempo das aulas na biblioteca. A reestruturação cognitiva é um processo que deve continuar intermitentemente durante todo o tratamento, sendo trabalho do psicólogo procurar informações que façam o indivíduo reavaliar seus pensamentos (TAYLOR, 2006; DIAS; CANAVEZ; MATOS, 2018). 
Ainda que Priscila tenha iniciado o processo de falar sobre o trauma, evidencia-se que existe ainda um caminho a ser percorrido antes do processo de alta. Em função de os atendimentos psicológicos em contexto de serviço-escola seguirem o calendário acadêmico, pode-se haver a necessidade de seguir as regras da instituição e da matriz curricular (CALDERONI, 1998) a qual, neste caso, impossibilita a permanência do mesmo estagiário por período maior de um ano. Sendo assim, a paciente foi encaminhada para outra estagiária, que dará continuidade ao processo psicoterapêutico no próximo ano.

\section{Conclusões}

A vivência de uma experiência traumática pode acarretar efeitos no desenvolvimento do sujeito, afetando-o em tarefas cotidianas, dificultando no estabelecimento de relações afetivas e sociais, assim como em seu funcionamento. O TEPT causa sofrimento psíquico, a partir do qual há necessidade de amparar, psicologicamente, o sujeito e fornecer-lhe instrumentos para que entenda os acontecimentos que vivenciou.

A TCC é apontada como uma das teorias mais eficazes na demonstração de resultados frente a pacientes com TEPT, uma vez que apresenta técnicas de exposição prolongada, utiliza técnicas de relaxamento e reestruturação cognitiva, a fim de diminuir o quadro ansioso, depressivo e os sentimentos relacionados ao transtorno e ao trauma. Aponta-se que apesar de ser um tema importante para discussão, destaca-se a necessidade de aprofundamento sobre o assunto, assim como a publicação de estudos que apontem para técnicas de intervenção.

Ainda que o tratamento psicoterápico tenha sido benéfico para Priscila, uma vez que pode iniciar o processo de ressignificar experiências associadas ao abuso, evidencia-se que é necessário dar continuidade no processo terapêutico, isso porque a paciente apresenta diferentes demandas referentes ao seu funcionamento e à forma como percebe o mundo. O local de laboratório de práticas em psicologia permitiu um lugar de fala para a paciente, a qual não era permitida ou autorizada pelos familiares a falar sobre o que aconteceu.

Fatores de limitação importantes de serem destacados são as delimitações relacionadas ao processo de testagem psicológica, cujo teste utilizado para mensurar questões associadas ao quadro depressivo, o Inventário Beck, é um instrumento de autorrelato, no qual a própria paciente assinala a pontuação das afirmações conforme sentiu-se nas últimas duas semanas, resultando, então, viés de resultado nas três vezes em que foi aplicado o teste.

Como fatores delimitadores desta pesquisa, aponta-se para a dificuldade de encontrar artigos científicos em período considerado recente, que tragam intervenções da TCC para a sintomatologia do TEPT. Ressalta-se, então, a necessidade de existirem maiores estudos sobre a temática, dado que cada paciente é único e possui necessidades diferentes a serem trabalhas em psicoterapia, sendo assim a publicação de artigos científicos só tem a contribuir para a Psicologia Clínica. 


\section{Referências:}

AMERICAN PSYCHIATRIC ASSOCIATION. Manual diagnóstico e estatístico de transtornos mentais (DSM-V). Porto Alegre: Artmed, 2014.

BECK, A. T.; STEER, R. A.; BROWN, G. K. BDI-II Manual. San Antonio: The Psychological Corporation. San Antonio, TX, 1996.

BECK, A. T.; STEER, R. A.; BROWN, G. K. BDI-II Manual. San Antonio: The Psychological Corporation. San Antonio, TX, 2011.

BECK, J. S. Terapia Cognitivo-Comportamental: teoria e prática, Porto Alegre: Artmed, 2013.

BRINO, R. F.; WILLIAMS, L. C. A. Concepções da professora acerca do abuso sexual infantile. Cadernos de Pesquisa, vol. 119, 113-128, 2003.

CALDERONI, M. L. O ato clínico de recepção e triagem. Percurso,10 (20), 150-155, 1998.

CLARK, D. A.; BECK, A. T. Terapia Cognitiva para os transtornos de ansiedade. Porto Alegre: Artmed, 2012.

COIMBRA, M. N. C. T.; MARTINS, A. M. O. O estudo de caso como abordagem metodológica no ensino superior. Nuances. São Paulo, 24(3), 31-46, 2013.

DE ANTONI, C.; KOLLER, S. H. Violência doméstica e comunitária. In CONTINI, M. L.F; KOLLER, S. H; BARROS, M. N. S. (Orgs.), Adolescência e psicologia: concepções, práticas e reflexões críticas Rio de Janeiro: Conselho Federal de Psicologia, pp. 85-91, 2002.

DENZIN, N. K.; LINCOLN, Y. S. Handbook of qualitative research. Thousand Oaks, Califórnia: Sage Publications, 2000.

DIAS, S. A.; CANAVEZ, L. S.; MATOS, E. S. Transtorno de Estresse Pós-Traumático em mulheres vítimas de violência doméstica: prejuízos cognitivos e formas de tratamento. Revista Valore, Volta Redonda, 3 (2): pag.597622, 2018.

DURAN, E. P. Eficácia da terapia cognitiva processual no transtorno de Estresse Pós-traumático. São Paulo, 2015. Dissertação (mestrado) - USP.

FLORENTINO, B. R. B. Abuso sexual, crianças e adolescentes: reflexões para o psicólogo que trabalha no CREAS. Rev. Psicol. vol. 26, no.1, Rio de Janeiro, 2014.

GADINI, B.; JÚNIOR, E.; FEIJÓ, M. Implicações do transtorno de estresse pós-traumático no trabalho: uma revisão bibliográfica narrativa. Psic., Saúde \& Doenças, vol. 19, no.3. Lisboa, 2018.

HABIGZANG, L. F.; CAMINHA, R. M. Abuso Sexual contra crianças e adolescentes: conceituação e intervenção clínica. São Paulo: Casa do Psicólogo, 2004.

HEFLIN, A. H.; DEBLINGER, E. Tratamento de um adolescente sobrevivente de abuso sexual na Infância. In REINECKE, M. A.. DATTILIO, F. M.; FREEMAN, A. (org.). Terapia Cognitiva com Crianças e Adolescentes: Manual para a prática clínica. Porto Alegre: Artmed, p. 161-178, 1999.

LUCÂNIA, E. R.; VALÉRIO, N. I; BARISON, S. Z. P; MIYAZAKI, M. C. O. S. Itervenção cognitivo-comportamental em violência sexual: estudo de caso. Psicologia em Estudo, v. 14, n. 4, p. 817-826, 2009.

MELLO, P. G; CAMINHA, R. M.; LORENZZONI, P.L; KRISTENSEN, C. Considerações clínicas sobre a psicoterapia cognitivo-comportamental de bancários com Transtorno de Estresse Pós-Traumático. Contextos Clínicos, vol.4, n.1, 2011

MONSON, C. M.; RESICK, P. A.; RIZVI, S. L. Transtorno de Estresse Pós-Traumático. In BARLOW, D. H (org). Manual Clínico dos Transtornos Psicológicos: tratamento passo a passo. Porto Alegre: Artmed, p. 62-113, 2016.

NATIONAL INSTITUTE OF CLINICAL EXCELLENCE. Post-traumatic stress disorder (PTSD): The treatment of PTSD in adults and children. London: Author, 2005.

OLIVEIRA, D. F. Aplicação da Terapia Cognitivo-Comportamental a pacientes com Transtorno de Estresse Pós-Traumático relacionado ao trabalho - revisão bibliográfica. São Paulo, 2017. Trabalho de Conclusão de Curso (especialização) - CETCC 
INTERVENÇÕES DA TERAPIA COGNITIVO-COMPORTAMENTAL (TCC) PARA TRANSTORNO DE ESTRESSE PÓS-TRAUMÁTICO: ESTUDO DE CASO

PADILHA, M. G. S.; GOMIDE, P. I. C. Descrição de um processo terapêutico em grupo para adolescentes vítimas de abuso sexual. Estudos de Psicologia, 9,53-6, 2004.

PARANHOS, M. E. Estudo de fidedignidade e validade do inventário de depressão de Beck (BDI-II) em adolescentes. Porto Alegre, 2009. Dissertação (mestrado) - PUC-RS.

PASSARELA, C. M.; MENDES, D. D.; MARI, J. J. Revisão sistemática para estudar a eficácia de terapia cognitivocomportamental para crianças e adolescentes abusadas sexualmente com transtorno de estresse pós traumático. Rev. Psiq. Clin. 37(2), 60-5, 2009.

POWERS, M.B; HALPERN, J. M; FERENSCHAK, M. P.; GILLIHAN, S. J.; FOA, E. B. A meta-analytic review of prolonged exposure for posttraumatic stress disorder. Clinical Psychology Review, 30, 635-641, 2010

RUGGIERO, K. J.; MCLLER, S. V.; DIXON, J. F. Sexual abuse characteristics associated with survivor psychopathology. Child Abuse \& Neglect, 24(7), 951- 964, 2000.

RUNYON, M. K.; KENNY, M. C. Relationship of attributional style, depression, and posttrauma distress among children who suffered physical or sexual abuse. Child Maltreatment, 7(3), 254-264, 2002.

SILVA, A. B. B. Mentes ansiosas: o medo e a ansiedade nossos de cada dia. São Paulo: Principium, 2017.

SILVA, M. A.; WENDT, G. W.; ARGIMON, I. I. Lilva, M. A., Wendt, G. W., Argimon, I. I. L. Inventário de depressão beck II: análises pela teoria do traço latente. Aval. Psicol. vol. 17, no. 3, Itatiba, 2018

SILVA, S. A.; HERZBERG, E.; MATOS, L. A. L. Características da inserção da psicologia nas pesquisas clínicoqualitativas: uma revisão. Bol. Psicol. vol.65, no.142, São Paulo, 2015.

SIQUEIRA, A. C.; ARPINI, S.; SAVEGNAGO, S. D. O. Família e abuso sexual na perspectiva de adolescentes em situação de vulnerabilidade social. Aletheia v. 34, p.109-122, 2011.

TAYLOR, S. Clinician's guide to PTSD: A cognitive-behavioral approach. New York: Guilford Press, 2006.

THE PSYCHOLOGICAL CORPORATION. Wechsler Abbreviated Scale of Intelligence Manual. San Antonio: Psychological Corporation, 1999

WECHSLER, D. Wechsler Abbreviated Scale of Intelligence Manual. San Antonio: Psychological Corporation, San Antonio: TX, 2014.

WHITBOURNE, S. K.; HALGIN. R. P. Psicopatologia - Perspectivas clínicas dos transtornos psicológicos. Porto Alegre: Artmed, 2015.

WRIGHT, J. H; BASCO, M. R.; THASE, M. E. Aprendendo a terapia cognitivo-comportamental: um guia ilustrado. Porto Alegre: Artmed, 2008.

YATES, D. B.; TRENTINI, C.M; TOSI, S. D.; CORRÊA, S. K; POGGERE, L.C.; VALLI, F. Apresentação da Escala de Inteligência Wechsler Abreviada (WASI). Avaliação Psicológica, 5(2), 227-233, 2006. 